\title{
El aprendizaje combinado y el desarrollo de las habilidades requeridas para la comunicación escrita
}

\author{
Combined Learning (face-to-face/virtual) and the Development of Written \\ Communication Skills
}

\author{
Olga Ligia Solano Córdoba ${ }^{1}$ \\ Instituto Tecnológico de Costa Rica \\ Cartago, Costa Rica \\ olsolano@itcr.ac.cr
}

Recibido 15 de abril de 2013 • Corregido 20 de mayo de 2013 • Aceptado 19 de junio de 2013

\begin{abstract}
Resumen. Este artículo resume los resultados del proyecto de investigación titulado El aprendizaje combinado (presencial y no presencial apoyado por el uso de internet) y su utilidad para el desarrollo de las habilidades de expresión escrita. Fue desarrollado durante los años 2011 y 2012 con el apoyo de la Vicerrectoría de Investigación del Instituto Tecnológico de Costa Rica. Se enmarca dentro de la investigación-acción: explora y sistematiza el aporte que brinda la implementación del aprendizaje combinado (cursos bimodales) para el desarrollo de las habilidades de expresión escrita. Se diseñaron estrategias didácticas para la utilización de foros, wikis, bitácoras electrónicas, gestores bibliográficos, software para elaboración de mapas conceptuales, así como correctores ortográficos y diccionarios en línea, como medios para fomentar el uso de la lengua escrita en contextos significativos. Estas se ejecutaron con un total de ochenta y un estudiantes de la Universidad de Costa Rica y del Instituto Tecnológico de Costa Rica y trece docentes (diez impartían lecciones en Educación Superior, dos en el nivel secundario, para el Ministerio de Educación Pública y una laboraba para ambos niveles). Posteriormente, se evaluaron los resultados a través del análisis de productos seleccionados, conversatorios con las docentes participantes, entrevistas a la población participante y análisis de las bitácoras de trabajo elaboradas por la investigadora. Se concluye que el aprendizaje combinado permite el desarrollo de las habilidades de expresión escrita, ya que esto implica el desarrollo de la capacidad y destreza para cumplir con las diferentes tareas del acceso al conocimiento sobre un tema para escribir, la planificación de los escritos, la producción textual propiamente dicha, la revisión del escrito y la divulgación de producto final.
\end{abstract}

Palabras claves. Aprendizaje combinado, habilidades para el desarrollo de la expresión escrita, escritura digital, escritura y TIC.

1 Doctora en Estudios Latinoamericanos de la Universidad Nacional de Costa Rica. Labora como docenteinvestigadora en la Escuela de Ciencias del Lenguaje del Instituto Tecnológico de Costa Rica y el Sistema de Estudios Generales de la Universidad de Costa Rica (Sede del Atlántico). Ha estado ligada al tema de Educación y Tecnología desde el año 1996. Durante 10 años laboró como Coordinadora Nacional del Programa de Informática Educativa de la Fundación Omar Dengo y el Ministerio de Educación Pública. Cuenta con experiencia como investigadora en el área de escritura y tecnologías de la información y la comunicación. Posee artículos publicados en esa línea y participa constantemente como ponente en Congresos Nacionales e Internacionales sobre esta temática. 
URL: http://www.una.ac.cr/educare

CORREO: educare@una.cr

\begin{abstract}
This paper summarizes the results of the research project El aprendizaje combinado (presencial y no presencial apoyado por el uso de internet) y su utilidad para el desarrollo de las habilidades de expresión escrita [The Use of Combined Learning (face-to-face and virtual) in Developing Written Expression Skills] conducted in 2011-2012 with the support of the Office of the Vice-President for Research of the Costa Rica Institute of Technology. This research-action study was focused on exploring and systematizing the contribution of combined learning activities (bimodal courses) to develop written expression skills. Didactic strategies were designed to use forums, wikis, blogs, bibliography managers, concept mapping software, as well as online spelling checkers and dictionaries as means to promote the use of written language in relevant contexts. The total study population included eighty-one students from the University of Costa Rica and the Costa Rica Institute of Technology, and thirteen faculty members (ten were higher education teachers, three were teachers of the Ministry of Public Education of Costa Rica working with students from the $7^{\text {th }}$ to the $11^{\text {th }}$ grades, and there was a teacher working in both contexts). The outcomes of the project were evaluated through the analysis of products selected, panel discussions with the participant teachers, interviews to the participant population, and the analysis of the researcher's project journals. This study concludes that combined learning contributes to improving written expression skills, since it involves the development of skills and competencies to accomplish different tasks related to the access of knowledge on a specific topic to be developed in writing, text planning, text production, proofreading and dissemination of the final product.
\end{abstract}

Keywords. Combined learning, developing writing skills, digital writing and TIC's.

En los años noventa, con la incorporación de las computadoras e internet en la vida cotidiana, se generaron nuevas prácticas en el uso de la lengua escrita. Hoy se navega por la red en busca de datos; se utilizan programas de edición de textos; se escriben correos electrónicos; se "habla", escribiendo en chats, a través del mesenger y de las redes sociales (Solano, 2010). Se ha señalado muchas veces que el uso de las tecnologías digitales ha contribuido al uso descuidado del idioma en su forma escrita; sin embargo, con la llegada a las plataformas virtuales y las posibilidades que brinda el aprendizaje combinado, la virtualidad se convierte en una valiosa oportunidad para fomentar el desarrollo de las habilidades escritas y constituye una posibilidad para retomar la necesidad de utilizar en forma adecuada (entiéndase de acuerdo con la norma), el uso del idioma en medios digitales.

Por otra parte, el uso de estas tecnologías se ha ido masificando y hoy es usada no solo por los nativos de la era digital, sino también por otras generaciones que -aunque con mayores dificultadesse han convertido en usuarias de estas, tal es el caso de las personas dedicadas a la docencia. Surge con ello la posibilidad de aprovechar la producción escrita que naturalmente se produce en estos soportes y convertirla en una oportunidad de reflexión sobre el uso del lenguaje y sobre la necesidad de utilizarlo correctamente (entiéndase de acuerdo con la norma) en los medios digitales.

En el caso del Instituto Tecnológico de Costa Rica (TEC) se cuenta, desde hace aproximadamente siete años, con una plataforma virtual cuyo objetivo es la integración de las tecnologías de información y comunicación a los procesos de enseñanza y aprendizaje. Asimismo, ha incentivado la implementación de cursos bimodales y cursos virtuales como parte de la oferta educativa de esta institución. En la misma línea han trabajado la Universidad de 
Costa Rica (UCR), a través de la Unidad de Apoyo a la Docencia Mediada con Tecnologías de la Información y la Comunicación (METICS), una unidad de la Vicerrectoría de Docencia. Además, con la llegada de la Web 2.0 (herramientas que permiten la publicación, edición y revisión de contenidos en la Red, por cualquier persona, aunque no sea especialista en informática) se han incrementado las posibilidades de implementar internet como medio para el aprendizaje, tanto para estudiantes universitarios como de secundaria.

Dado lo anterior, este artículo de investigación ofrece insumos para la implementación del aprendizaje combinado y su utilidad para el desarrollo de las habilidades de escritura.

\section{Antecedentes}

Sánchez (2005) señala que hay cuatro estudios que pueden servir de base para determinar el diagnóstico de los problemas de expresión escrita del estudiantado costarricense de secundaria y universidad. Estos fueron elaborados por Jiménez (1987); Rodino y Ross (1985); Rojas (1987); Rojas, Porras, y Umaña (1986); quienes, en sus investigaciones, trabajaron con estudiantes universitarios de la Universidad Estatal a Distancia (UNED) y de la Universidad de Costa Rica (Sede de Occidente) y con estudiantes que estaban cursando el último año de la educación secundaria. Llegaron a las siguientes conclusiones:

- Los estudiantes muestran problemas para diferenciar elementos que pertenecen al registro oral o al registro escrito. Cuentan con poco conocimiento (o ninguno) de los parámetros de construcción de los textos escritos, por lo cual escriben igual a como hablan.

- Se subutilizan los recursos gramaticales del idioma, hay poca variedad léxica, el uso de la puntuación es descuidada, se limita al uso de pocos signos y se desconocen usos básicos.

- Los textos son incompletos, no desarrollan apropiadamente el mensaje, no hay adecuada conexión entre las ideas y no se refleja planeamiento.

- Las redacciones evidencian interés solo por la inmediatez del texto.

- Hay exceso de subordinación, pobreza de ideas, poca variedad y mucha repetición.

- Alteración del orden lógico y sintáctico de la estructura oracional.

- Desajuste temático entre el título y el contenido.

- Desconocimiento del uso del párrafo.

- Repetición de palabras, barbarismos, cosismos, alguismos, oseismos, etceterismos, verbos fáciles, queísmos y falta de sinónimos. 
URL: http://www.una.ac.cr/educare

CORREO: educare@una.cr

La crítica fundamental que plantea Sánchez (2005) a los estudios señalados es que:

No se han abordado las deficiencias desde una perspectiva discursiva que permita evaluar el problema en su dimensión textual, no en su microcomposición al analizar elementos aislados; es decir, no se han estudiado los problemas en el empleo de los elementos lingüísticos dentro de la totalidad del texto, sino separados unos de otros, lo que no posibilita darse una idea de la calidad de los textos en cuanto tales .... (p. 269)

Precisamente esa visión de análisis del problema de la escritura no es aislada, responde al enfoque que ha privado en su enseñanza, un enfoque centrado en la gramática: aprendizaje de reglas ortográficas, morfosintaxis y léxico (Solano, 2010).

En relación con el tema de la escritura y las tecnologías de la información y comunicación, Goldberg y Cook, citados por Henao (2006) plantean que:

Un meta-análisis de las investigaciones realizadas entre 1992 y 2002 indica que, cuando los estudiantes de educación básica escriben utilizando procesadores de texto, tienden a producir textos más extensos, efectúan más cambios en los borradores de sus escritos y elaboran composiciones de mejor calidad, que cuando escriben con lápiz y papel. Este efecto tiende a ser mayor en los estudiantes de los grados medios y superiores. (p. 74)

Henao (2006) reseña algunas investigaciones realizadas en la Universidad de Antioquía por el Grupo didáctica y nuevas tecnologías, desarrolladas con estudiantes de educación general básica, las cuales se refieren al impacto de las tecnologías de la información y la comunicación en la enseñanza de la lecto-escritura. Las conclusiones a las que llegan esas investigaciones se resumen a continuación:

- Los textos que produjeron los alumnos en el computador revelaron mejor calidad literaria, mayor longitud, mejor nivel de adjetivación y mayor diversidad léxica que los textos elaborados manualmente.

- La población participante, al verse liberada de las exigencias psicomotrices de la escritura manual, centraban más su atención en el contenido.

- El computador permitió, a la población participante, un ejercicio de escritura menos restrictivo y más propicio para la creatividad.

- La facilidad de revisión que ofrece el procesador de textos les motivó a no resignarse con el primer borrador y a buscar formas alternativas de expresar sus ideas. 
- El uso de un entorno para la comunicación escrita, apoyado en el correo electrónico y otras herramientas informáticas, impactó positivamente el valor, el sentido y la funcionalidad que los participantes le asignan a la escritura, lo cual se notó en la valoración de la escritura como herramienta de expresión y comunicación, un mayor dominio del tema, la comunicación adecuada de ideas, el ver la escritura como una posibilidad de éxito profesional y asumirla la escritura como una herramienta útil más allá del contexto escolar.

- Cuando se escribe con una herramienta hipermedial se realizan actividades de escritura más extensas y se mantiene la motivación por la escritura.

- El estudiantado revisa más y con mayor independencia sus textos cuando utilizan una herramienta como hipermedia, lo cual trae consigo un mejoramiento en la calidad de sus procesos textuales.

- Los trabajos realizados mostraron rigor y precisión conceptual, fuerza descriptiva, argumentativa e ilustrativa y un nivel de creatividad superior.

- El estudiantado, sin necesidad de instrucción directa, puede lograr un nivel de competencia en el uso de diversas herramientas informáticas.

En Costa Rica y aunque la utilización de la computadora e internet en ambientes educativos es de larga data, solamente se encontró una investigación relacionada con el tema. Argüello, Mora y Quirós (2012) trabajaron el desarrollo de las habilidades de escritura en estudiantes del III ciclo de la Educación General Básica por medio de talleres para la construcción de párrafos expositivos, narrativos y descriptivos con utilización de las TIC. Llegaron a concluir que estas permiten el desarrollo de la competencia escritora del alumnado, ya que estos recursos constituyen medios que se ajustan a las necesidades e intereses de las personas llamadas nativas digitales y que, gracias a la inclusión de esas tecnologías, los sujetos de investigación accedieron con mayor optimismo a actividades tales como la corrección, sobre todo si se realiza en forma digital y no siguiendo el procedimiento tradicional con elementos analógicos. Además, el uso de las TIC medió en forma positiva para facilitar un aprendizaje constructivista y cooperativo.

Dado lo anterior, se puede afirmar que la investigación sobre TIC y escritura es aún incipiente en Costa Rica, lo cual representa una excelente oportunidad para que la Escuela de Ciencias del Lenguaje del Instituto Tecnológico de Costa Rica contribuya a la realización de proyectos de investigación que generen insumos para la implementación de la virtualidad en el aprendizaje de campos específicos, tales como el desarrollo de las habilidades de escritura. Por ello, esta propuesta busca responder a la siguiente pregunta de investigación:

¿Cuáles son los beneficios que ofrece el aprendizaje combinado para el desarrollo de las habilidades de expresión escrita? 
URL: http://www.una.ac.cr/educare

CORREO: educare@una.cr

\section{Referentes conceptuales}

\section{El aprendizaje combinado}

El aprendizaje combinado es el término que se usa para referirse a aquel modo de aprender que combina la enseñanza presencial con la tecnología no presencial (Coaten, 2003; Marsh II, McFadden y Price, 2003). Utiliza diversas herramientas, como: uso de software, recursos en web y prácticas de gestión del conocimiento, incluyendo clases presenciales (cara a cara) y el aprendizaje a ritmo individual.

Las características de este modo de aprender son las siguientes:

- Presencia y guía directa del docente.

- Inclusión de actividades propias de la virtualidad: foros, conversaciones electrónicas, bitácoras electrónicas, uso de wikis, acceso a bases de datos, videoconferencias, etcétera.

- Utilización de diversas técnicas y metodologías de enseñanza.

- Fomento del aprendizaje colaborativo.

Esta metodología puede aplicarse utilizando las plataformas virtuales de aprendizaje disponibles en muchos centros de estudio y a través de las herramientas de la Web 2.0, tales como foros electrónicos, bitácoras electrónicas, wikis, software de uso libre como el "C Map Tools", motores de búsqueda y gestores bibliográficos.

\section{Enseñanza y aprendizaje de la escritura}

Álvarez y Ramírez (2006) recogen y analizan las teorías o modelos de escritura predominantes en la investigación educativa sobre comprensión y producción de textos. Analizan los modelos de Bathia (1999); Beaugrande y Dressler (1982); Bereiter y Scardamalia (1982); Candlin y Hyland (1999); Coulthard (1994); Grabe y Kaplan (1996); Grupo Didactext (2003); Hayes y Flower (1980); Nystrand (1982); Sinclair (1994 y concluyen:

En su conjunto, los modelos propuestos presentan convergencias muy importantes, tales como: i) ponen de manifiesto la importancia del contexto en el que se realiza la tarea (intenciones, destinatarios, tipo de texto, etc.); ii) resaltan el desarrollo de estrategias cognitivas y metacognitivas para la producción textual; iii) destacan la diferencia entre la producción oral y la producción escrita; iv) asumen la escritura como un proceso complejo que requiere de entrenamiento constante; v) hacen hincapié en el proceso más que en el producto; vi) señalan unas etapas o fases fundamentales para la construcción del texto 
URL: http://www.una.ac.cr/educare CORREO: educare@una.cr

(planificación, textualización, revisión y edición) y vii) insisten de manera particular en la revisión, tanto desde el punto de vista del proceso, como del producto final, que es el texto. (Álvarez, y Ramírez, 2006, p. 57)

A partir de los insumos que brinda el análisis hecho por los autores mencionados y para efectos de esta investigación, se entenderá la escritura como un proceso que consta de varias etapas y que requiere que la persona que escribe cuente con capacidad y destreza para:

- Seleccionar información confiable en función de un tema, la intención y el público.

- Clasificar, integrar, generalizar y jerarquizar la información seleccionada.

- Interpretar la información.

- Desarrollar esquemas estableciendo relaciones entre la información seleccionada.

- Valorar la información seleccionada.

- Conformar una opinión.

- Plantear nuevas ideas.

- Argumentar las ideas.

- Ser coherente.

- Usar el registro adecuado según el tema, la intención y el público destinatario.

- Elaborar borradores o textos intermedios.

- Identificar faltas orto-tipográficas, faltas gramaticales.

- Usar adecuadamente el discurso ajeno en el discurso propio.

- Usar correctamente, entiéndase de acuerdo con la norma, el idioma.

- Utilizar referencias.

- Generar nuevas ideas y nuevos problemas sobre los cuales escribir.

\section{Internet y las posibilidades que brinda para el desarrollo de las habilidades requeridas para la comunicación escrita}

Si se considera, según se ha mencionado anteriormente, la escritura como un macroproceso y se retoman las cuatro fases propuestas por el Grupo Didactex, se va a encontrar en Internet una serie de herramientas que pueden ayudar a potenciar el desarrollo de las habilidades de expresión escrita, a través de la implementación del aprendizaje combinado. Al respecto, 
URL: http://www.una.ac.cr/educare

CORREO: educare@una.cr

Tinajero (2006) plantea que ". . . internet y las computadoras en educación, ... nos sirven para mediar, ordenar y posicionar la información para facilitar el desarrollo del lenguaje sobre el cual construimos los conocimientos..." (p. 103).

A continuación se hace mención de las herramientas que se utilizaron en esta investigación, como medios para apoyar el desarrollo de las habilidades requeridas para la comunicación escrita:

\section{Los motores de búsqueda}

Son programas instalados en un servidor conectado a internet. Nacieron de la necesidad de organizar la información anárquica contenida en la red. Es necesario aprender a detallar los pedidos de información con la precisión necesaria para que el mecanismo de búsqueda brinde la menor cantidad posible de opciones.

\section{EI C Map Tools}

Es un software que permite la elaboración de mapas conceptuales (representación de relaciones entre conceptos) de manera rápida y fácil.

\section{Las bitácoras electrónicas}

Es un espacio personal en Internet, que se actualiza periódicamente, recopila cronológicamente textos o artículos, donde el más reciente aparece primero. Permite la participación de terceros a través de comentarios. También se le conoce como "blogs" y existen de tipo personal, periodístico, empresarial, corporativo, tecnológico y educativo, entre otros.

\section{El foro electrónico}

Es una discusión, a través de un sitio de Internet, sobre una determinada temática (formulada con anticipación). Las personas participantes deben ingresar a la dirección electrónica indicada y después de leer las indicaciones dadas por quien modera, deben responder por escrito a una pregunta generadora (formulada por quien modera) que da inicio a la discusión del tema. Es asincrónico, lo que significa que las participaciones pueden hacerse en cualquier momento, durante el período de duración del foro.

\section{Los wikis}

Son una aplicación que permite visualizar, en línea, un conjunto de páginas perteneciente a un grupo de personas, las cuales a través de ese espacio añaden contenidos y editan los existentes. 


\section{Los gestores de referencias bibliográficas}

Son herramientas que permiten manejar bases de datos de referencias bibliográficas obtenidas a partir de distintas fuentes. Estos crean, mantienen, organizan, y dan forma a las referencias bibliográficas de acuerdo con diferentes estilos de citación (Vancouver, Ansi, APA). Existen gestores bibliográficos de uso gratuito y también gestores que requieren de pago.

\section{Metodología}

\section{Diseño de investigación}

El diseño utilizado para el desarrollo de esta investigación es conocido como investigaciónacción. Sandín (2003) plantea que la investigación-acción construye el conocimiento por medio de la práctica.

\section{Sujetos participantes}

Se contó con la participación de 13 docentes: tres que laboraban en el Sistema de Estudios Generales de la Sede del Atlántico de la Universidad de Costa Rica (dos impartían lecciones de comunicación y una impartía lecciones de filosofía), tres que laboraban como profesoras de español en colegios del Ministerio de Educación Pública (dos en el Liceo de Moravia perteneciente a la provincia de San José y otra en el Colegio de Dulce Nombre, de la provincia de Cartago), seis docentes que laboraban en la Escuela de Ciencias del Lenguaje del Instituto Tecnológico de Costa Rica (cuatro impartían lecciones de inglés, dos de comunicación) y una docente que laboraba en la Escuela de Ingeniería Agrícola del Instituto Tecnológico de Costa Rica. Estas docentes manifestaron interés en participar en esta experiencia, dado que deseaban implementar el uso de herramientas computacionales en sus labores docentes y nunca habían utilizado la metodología de aprendizaje combinado como parte de su labor.

También participaron un total de ochenta y un estudiantes ( 22 del grupo 01 del curso Cl-5107-Seminario de Investigación en el Instituto Tecnológico de Costa Rica; 27 del grupo 22 del curso EG-0125-Humanidades, en el recinto de Paraíso de la Sede del Atlántico de la Universidad de Costa Rica; 12 del grupo 32 del curso EG-0125-Humanidades y 20 del curso RP-1203Redacción y Ortografía), estos dos últimos cursos se impartían en el Recinto de Guápiles de la Sede del Atlántico de la Universidad de Costa Rica. 
URL: http://www.una.ac.cr/educare

CORREO: educare@una.cr

\section{Plan de acción}

Estuvo conformado por tres etapas:

1. Diseño de estrategias para el desarrollo de las habilidades de expresión escrita a partir de la implementación del aprendizaje combinado.

2. Implementación de las estrategias diseñadas con las poblaciones seleccionadas.

3. Evaluación de las estrategias implementadas.

\section{Instrumentos de recolección de la información}

Para la recolección de información se utilizaron los siguientes instrumentos:

- Bitácoras de trabajo elaboradas por la investigadora durante el desarrollo de la investigación.

- Entrevistas a profundidad. Se realizaron entrevistas a profundidad a cinco docentes que participaron en el curso El aprendizaje combinado y las posibilidades que brinda para el desarrollo de las habilidades de escritura y a 5 estudiantes del curso Cl-5107 Seminario de Investigación.

- Entrevistas grupales a 12 de los estudiantes del curso EG-0124 Comunicación del bloque de humanidades. Grupo 22 (en cada entrevista grupal se contó con 6 estudiantes) que participaron en el foro electrónico "Ciencia-Ficción y Posibles Futuros". Las entrevistas se grabaron y luego se transcribieron para su análisis.

- Bitácoras de análisis de productos elaborados por la población que participó en la investigación.

- Grabación de la segunda sesión presencial del curso dirigido a docentes (se formuló como conversatorio).

\section{Análisis de la información}

Para el análisis de la información se transcribieron las entrevistas y el conversatorio, posteriormente se analizaron para determinar la forma en que las estrategias implementadas contribuyeron al desarrollo de las habilidades de expresión escrita de las personas participantes. Para esto se elaboraron matrices y se extrajeron citas en las cuales se hacía referencia al aporte que les brindaron las estrategias implementadas para el desarrollo del proceso de escritura. 
Se seleccionaron, al azar, productos elaborados (muestras de los foros, bitácoras, wikis, mapas conceptuales) por las personas participantes para analizar y valorar el desempeño y progreso en el área de la expresión escrita.

También se analizaron las bitácoras que la investigadora había ido realizando a lo largo de la investigación.

Finalmente se trianguló la información obtenida de las tres fuentes de información señaladas anteriormente.

\section{Resultados obtenidos}

El 85\% de las docentes participantes en esta experiencia no habían tenido la oportunidad de participar en el curso impartido con la modalidad de aprendizaje combinado. Ellas demandaron mayor cantidad de apoyo presencial durante las sesiones virtuales que los grupos de estudiantes.

En general las dificultades que se presentaron fueron por falta de atención al leer las instrucciones de los materiales y, por consiguiente, por no ejecutarlas, según se señalaba. Sin embargo, conforme avanzó el curso, se adquirió conciencia de la importancia de seguir al pie de la letra las instrucciones recibidas.

Tanto la investigadora como la asistente del proyecto daban apoyo (presencial y virtual) a las docentes que participaban en esta experiencia. Es interesante resaltar, sin embargo, que la mayor cantidad de apoyo se dio por parte de las participantes que estaban más cerca de la investigadora y de la asistente (compartían el mismo lugar de trabajo) y fue menor por parte de las participantes que laboraban en otras instituciones o bien en la misma institución, pero en otra unidad académica. Podría pensarse que la facilidad de contar con el apoyo presencial fue la causa de una mayor demanda y no necesariamente que no pudieran realizar las actividades de aprendizaje con el uso de los medios disponibles (manuales, indicaciones por escrito, sesiones virtuales).

Con respecto a los materiales elaborados para las sesiones virtuales, las docentes participantes consideraron que estaban bien redactados y constituían una excelente guía para el aprendizaje de las herramientas.

En general el tiempo utilizado por las docentes participantes para la realización de las actividades correspondió al planificado. Solamente en un caso hubo que dar tiempo extra (más allá de finalizado el curso) para que una de las docentes participantes cumpliera con el mínimo esperado para la aprobación del curso.

Las docentes participantes manifestaban diferentes niveles de conocimiento y destreza para el manejo de herramientas computacionales. El aprendizaje combinado es una modalidad de aprendizaje que permite respetar el ritmo de aprendizaje. El hecho de que cada quien pudiera avanzar a su propio ritmo, fue una de los aspectos más valorados por las participantes. 
URL: http://www.una.ac.cr/educare

CORREO: educare@una.cr

El estudiantado que participó en esta experiencia tampoco había vivenciado anteriormente esta modalidad de aprendizaje. A la hora de planteárselo, les pareció interesante y novedoso. Asumieron el reto, pero antes se les debió aclarar dudas en cuanto a la evaluación y manifestaron temor por los problemas que podría traer el contar con dificultades para el acceso a internet o a la plataforma virtual que se utilizaría (en el caso del TEC que se utilizó el TEC DIGITAL como entorno virtual de aprendizaje). En general, las experiencias les parecieron interesantes, entretenidas y accesibles.

Las estrategias diseñadas para apoyar el desarrollo de las habilidades de expresión escrita a través de la implementación del aprendizaje combinado fueron las siguientes:

\section{Aprendo a discriminar información en Internet}

Internet constituye hoy una de las opciones más utilizadas para la búsqueda de información sobre un tema. Sin embargo, el estudiantado desconoce criterios para seleccionar información confiable en Internet. Es necesario dotarlo de la información necesaria para realizar búsquedas eficientes y para que cuente con criterios para discriminar información. Esto implica ofrecerle recomendaciones para el uso de los buscadores: identificación de conceptos y palabras claves, uso de los operadores boléanos para precisar las búsquedas, uso de búsquedas avanzadas, uso de google académico. El docente o la docente, después de desarrollar los contenidos señalados anteriormente, puede seleccionar una lista de páginas en internet y solicitar evaluar la confiabilidad de esas páginas, a partir de criterios establecidos para ello.

El 100\% del estudiantado que ejecutó esta estrategia manifestó que hasta ese momento no conocían criterios para seleccionar información confiable en Internet, desconocían el uso de google académico y tampoco conocían los operadores boleanos. Consideraron que esta sesión había sido muy valiosa, ya que anteriormente sus docentes, les manifestaban que no todo lo que existía en Internet era valioso, pero desconocían cómo identificar lo confiable de lo no confiable y, sobre todo, usaban material de fuentes como el rincón del vago y Wikipedia, sin saber que estos sitios no eran garantía de calidad. Sí sabían de la existencia de google, pero no tenían herramientas para discriminar la información encontrada y el $97 \%$ desconocía la existencia de google académico.

\section{Sintetizo una lectura o una conferencia utilizando el C Map Tools}

Un mapa conceptual es una forma de representar esquemáticamente un conjunto de significados conceptuales. Es un valioso instrumento para visualizar conceptos y relaciones jerárquicas entre los conceptos. Constituye una valiosa ayuda para extraer conceptos, a partir de lecturas y para establecer relaciones entre esos conceptos. También puede servir para establecer los conocimientos que se tienen sobre un tema que se va a investigar. El C Map 
TOOLS es un software gratuito para la elaboración electrónica de mapas conceptuales (cuenta con manual para su uso que puede ser consultado a través de Internet).

Se puede solicitar al estudiantado elaborar mapas conceptuales, utilizando el CMap Tools sobre las lecturas realizadas de un tema de interés. También se les puede solicitar elaborar un mapa conceptual para planificar un escrito.

Con respecto al uso del C Map Tools, la mayoría de los estudiantes no presentaron dificultades para la descarga y el uso del software. Los pocos que tuvieron las resolvieron por su cuenta, preguntando a un profesor de la especialidad o a otra de las personas que integraban el grupo. Este aspecto es muy importante, pues de forma natural se gestó el aprendizaje colaborativo y el autoaprendizaje. De las conversaciones y entrevistas realizadas con las poblaciones participantes se pudo determinar que solo el 3,33\% del estudiantado manifestó conocer este software con anterioridad, el 100\% valoró muy bien la herramienta y la consideraron útil para facilitar el proceso de síntesis y compresión de textos estudiados.

En el caso de las docentes, el 100\% desconocía la herramienta y luego de utilizarla consideraron que era una valiosa herramienta para la comprensión de un texto.

\section{Leo, pienso, opino e interactúo a través de foros electrónicos}

Esta estrategia puede aplicarse a partir de la lectura de una obra literaria. Se puede invitar al autor de la obra (si es contemporánea) a conversar electrónicamente con el estudiantado que participó en esa lectura. Si se trata de una obra literaria cuyo autor o autora ya falleció, se puede invitar a académicos que hayan estudiado esta obra. También se puede generar la conversación electrónica entre estudiantes de diferentes lugares que también estén leyendo o hayan leído la obra. Otra posibilidad es generar foros electrónicos a partir de cualquier lectura (aunque no sea literaria). También pueden realizarse foros electrónicos a partir del visionado de una película. El personal docente a cargo de la actividad puede contactar personas conocedoras del tema en estudio para generar el intercambio correspondiente.

Las poblaciones de estudiantes participantes manifestaron que el foro electrónico les había parecido una experiencia interesante, entretenida y accesible. Dijeron “la opinión que uno tiene puede enriquecerse con la de expertos en la materia y con la de los mismos compañeros que ayudan a ver otro punto de vista". Esto porque en algunas ocasiones se planteó una pregunta y no necesariamente la contestaron los autores, sino otros compañeros. Consideraron que a través del foro se pueden detectar los puntos en común, ya que "hubo muchas coincidencias en lo que uno sintió cuando leyó el cuento". Se valoró mucho la posibilidad que brinda un foro electrónico para la interacción grupal, ya que "a través del foro se puede conocer la opinión de muchas personas, sin necesidad de estar todos en la clase o ser compañeros, en el foro se pudo 
URL: http://www.una.ac.cr/educare

CORREO: educare@una.cr

intercambiar con los autores del libro". Expresaron que, si el foro hubiera sido presencial, no hubieran tenido la oportunidad de plantear tantas ideas como lo hicieron en el foro virtual, "ya que hubo varios días para pensar en lo que se quería plantear, hubo más tiempo para plantear las ideas, para pensarlas, analizarlas y no preguntar cualquier cosa". Consideraron que hubo más posibilidades de participación, puesto que en un foro presencial "la gente no se anima a preguntar por vergüenza, en el foro electrónico no da vergüenza", además, dijeron que "en un foro electrónico hay más tiempo para elaborar las participaciones", "se puede ingresar en cualquier momento y solo se escribe, uno no está viendo a las personas, se le quita la pena de preguntar, uno nada más escribe lo que piensa".

Con respecto al proceso de elaboración de las participaciones, manifestaron que escribieron lo que sintieron y lo que se les iba ocurriendo, pero luego revisaban lo que habían escrito. A algunos les dio mucho miedo escribir, otros manifestaron "que pensaron mucho lo que iban a decir, lo leían varias veces, como tres veces, para no expresar incoherencias". En general manifestaron que escribían varias veces lo que iban a decir, lo borraban y no lo enviaban hasta que estuvieran seguros. Otra participante manifestó que leía todos los comentarios y luego formulaba sus participaciones y se apoyaba en la de sus compañeros. Algunos las escribían en "Word" y otros las escribían de una vez en el foro, pero no las enviaban hasta que estaban seguros de que no tenían errores ortográficos y que habían formulado correctamente sus ideas.

En general, consideraron que la actividad les ayudó a comprender el libro y a enriquecer la lectura. La interacción con los autores y las autoras les pareció muy enriquecedora e interesante.

Las participaciones hechas por estudiantes del grupo 22 del Bloque de Humanidades tienen desde dos líneas (la minoría) hasta de 16. La mayoría con 6 a 11 líneas. En el caso de los estudiantes del Seminario de Investigación, las participaciones la extensión poseía al menos dos párrafos de 10 líneas aproximadamente. En ambos casos las participaciones tuvieron pocos errores ortográficos y reflejaban que habían leído los materiales asignados para la participación en el foro.

En cuanto a las docentes, las participaciones en el foro superaron las solicitadas (3 por persona) y la herramienta les permitió conversar extensamente sobre las temáticas abordadas. La mayoría de las participaciones tenían entre 10 y 20 líneas, algunas pocas fueron de 5 líneas. Las participantes planteaban sus puntos de vista y los sustentaban satisfactoriamente, las participaciones eran claras, se generó el debate y el intercambio de opiniones.

La herramienta del foro constituyó un instrumento para el intercambio y cumplió muy bien su cometido. Los foros contribuyeron a conformar un ambiente de trabajo en equipo, pues, aunque no se vieran presencialmente, se sintieron acompañadas en la virtualidad.

Se señaló que un acierto fue la claridad de las indicaciones: definición del mínimo de participaciones por hacer y el tipo de participaciones (era obligado comentar o refutar 
participaciones hechas y hacer participaciones nuevas); además de la labor de moderación, la cual fue indispensable para el éxito de la experiencia.

\section{Interactúo a través de los wikis}

Los wikis pueden servir para la organización de actividades por ejecutar en grupo: ensayos grupales, investigaciones grupales u organización de eventos. La herramienta permite que sea utilizada por un máximo de cuatro personas. A través esta herramienta se pueden desarrollar trabajos para realizar fuera del horario escolar (trabajo extra-clase).

La herramienta fue muy bien valorada y el uso de esta no causó dificultad entre las docentes participantes. En la página del wiki dedicada a plantear las formas en que se puede aplicar lo aprendido, se señaló concurrentemente las posibilidades que brinda para potenciar el trabajo colaborativo en el área de escritura. También se valoró el hecho de que elimina el inconveniente de tener varias versiones del mismo documento. Para el total de docentes que participaron en el curso esta herramienta era desconocida. Según el seguimiento dado, una vez finalizado el curso, al menos una de las docentes participantes hizo uso de esta herramienta como apoyo a sus actividades educativas.

Esta estrategia también se implementó con dos grupos de 6 estudiantes que estaban realizando su proyecto de investigación para el curso Taller de Investigación. A este grupo de estudiantes se les dio un taller de 60 minutos para el uso de la herramienta. Finalizado el taller, los equipos de trabajo se dedicaron a construir el wiki y a utilizarlo para escribir la "tesina" (un trabajo de investigación con el que tienen que cumplir como parte de sus tareas para aprobar humanidades). La herramienta fue utilizada durante todo el semestre, la profesora tutora de la investigación revisaba semanalmente los avances de la investigación.

Con respecto a esta herramienta, el estudiantado consideró que fue un acierto su uso debido a que el trabajo se modificaba y aparecía actualizado inmediatamente, no gastaban papel, todo el grupo tenía acceso a la última versión, no tenían que reunirse físicamente, todo el grupo conocía el trabajo en su totalidad y podían aportar en cualquier momento

El estudiantado que pertenecía al Recinto de Guápiles se mostró complacido con el uso de la herramienta; sin embargo, por falta de acceso a internet en sus casas, lo utilizaban sobre todo en la universidad y lo hacían todos juntos, en grupo. En pocas ocasiones trabajaron independientemente. Este grupo sobre todo valoró que para la presentación final del trabajo de investigación solo tenían que copiarlo del wiki y transcribirlo en documento Word.

El análisis de proceso de elaboración del trabajo de investigación (el wiki tiene la página de cambios recientes en la cual el personal docente puede evaluar el trabajo de cada integrante del equipo, ya que ahí quedan registradas todas las participaciones) refleja que todas las personas que integraban los equipos habían participado en la elaboración de la investigación. 
URL: http://www.una.ac.cr/educare

CORREO: educare@una.cr

Se notaba cómo todo el equipo participaba tanto en el desarrollo de los contenidos como en corregir aspectos de redacción y ortografía. Las ideas planteadas se argumentaban satisfactoriamente y en este proceso intervenía todo el equipo.

\section{Aprovecho los gestores bibliográficos, una de las posibilidades que brinda Internet para el desarrollo de la investigación}

Existen gestores bibliográficos de uso gratuito y otros que requieren de pago por su uso. Zotero es un gestor gratuito, se puede localizar en la Web, a través de un buscador. Ahí aparecen las indicaciones para bajar el software, así como manuales para su uso. Este permite a sus usuarios y usuarias recolectar, administrar y citar investigaciones. Se usa para administrar bibliografías y referencias al escribir ensayos y artículos. El procesador de palabras Word cuenta con un gestor bibliográfico. En Costa Rica un grupo de estudiantes del Colegio Científico de Pérez Zeledón presentó, como proyecto de una feria científica, el gestor llamado Tupera. Este gestor ha sido mejorado en el transcurso de los años y hoy constituye una valiosa herramienta para gestionar referencias bibliográficas. En internet pueden encontrarse tutoriales para aprender a utilizar cualquiera de los tres gestores señalados anteriormente. El personal docente puede conformar grupos de dos o tres estudiantes y solicitarles que localicen en internet, un tutorial que les permita aprender el uso de cualquiera de los tres gestores señalados anteriormente. Una vez que lo hayan aprendido a utilizar, podrán crear bibliografías utilizando cualquiera de ellos.

Esta herramienta resultó totalmente desconocida para el 100\% de las docentes participantes y no tuvieron dificultades en utilizarla. En realidad, la mayor dificultad consistió en poder hacer la tarea con todas las demandas propias de un cierre de curso (esta fue la última actividad planteada y coincidió con el cierre de curso lectivo). Resultó una herramienta muy bien valorada, según manifestaron muy apropiada para ponerla en práctica tanto para cumplir con actividades propias de un docente (manipular la biografía de un artículo científico en proceso de escritura, de una tesis para obtener un grado académico así como para trabajarla con estudiantes de los diferentes cursos que impartían las docentes participantes, tanto en secundaria como universitaria.

El $100 \%$ del estudiantado participante también desconocía esta herramienta. Tampoco tuvieron dificultad en aprender su uso y manifestaron que era lamentable no haberla conocido antes, pues hubiera sido de gran ayuda para la realización de trabajos de investigación. 
URL: http://www.una.ac.cr/educare CORREO: educare@una.cr

\section{Divulgo e intercambio conocimientos usando las bitácoras electrónicas}

Es necesario que los productos escritos que se elaboren no solo sirvan para cumplir con una tarea escolar. Por esta razón las bitácoras electrónicas se convierten en una valiosa herramienta para generar procesos de escritura auténticos.

El personal docente puede solicitar al estudiantado que ingresen a www.blogger.com y exploren el uso de esta herramienta. Esta puede ser utilizada en cualquiera de las etapas del proceso escritura. Se le puede pedir al estudiantado que elaboren bitácoras electrónicas para divulgar sus productos escritos. Se puede contactar a personas especialistas en los temas que se desarrollan en las bitácoras para que puedan retroalimentar el trabajo elaborado. Asimismo, se le puede asignar a cada estudiante la lectura y evaluación de otras bitácoras (elaboradas por integrantes del grupo).

Un 20\% del personal docente participante tenía un conocimiento muy básico del manejo de la herramienta. Con la ayuda de una guía para la elaboración de bitácoras electrónicas (esta fue elaborada para efectos de este curso, y para ello se pusieron en práctica las consignas ya mencionadas para las otras guías), cada participante construyó su bitácora y la compartió con sus colegas. Simultáneamente se desarrolló el foro Compartiendo experiencias y reflexiones sobre los blogs como herramienta educativa. En este se intercambiaron las experiencias generadas a partir de la elaboración de la bitácora y también se comentaron las lecturas asignadas para esta temática. Con respecto al uso de las bitácoras se puede afirmar que esta herramienta fue muy bien valorada por las participantes, no tuvieron dificultad para elaborar su bitácora y las participaciones en el foro reflejaron la apropiación que hicieron de esta como una herramienta educativa.

El $80 \%$ del estudiantado participante desconocía el uso de esta herramienta. Un $40 \%$ de la población utilizaba poco la computadora, este grupo iba más lento y llegaron a utilizar en un $50 \%$ las posibilidades que brinda la herramienta. Cada cual avanzó a su ritmo, para algunas personas fue más fácil que para otras. Influyó mucho la familiaridad que tuvieran con el uso de computadoras.

El estudiantado se manifestó muy complacido de utilizar esta herramienta, les pareció más dinámico escribir en la computadora que en un cuaderno, además de que podían incluir imágenes, videos y establecer enlaces con otras páginas relacionadas con los productos desarrollados en el taller (diccionarios electrónicos y páginas web). 
URL: http://www.una.ac.cr/educare

CORREO: educare@una.cr

\section{Reviso y corrijo mis productos escritos utilizando los correctores ortográficos y los diccionarios en línea}

Para el proceso de revisión y edición de un texto contamos con diccionarios en línea y correctores ortográficos. Un corrector ortográfico es una herramienta que detecta faltas ortográficas y permite corregirlas de forma automática o manual. Tenemos el diccionario de la Real Academia Española en línea, así como diccionarios de sinónimos y antónimos. El personal docente puede pedir a sus estudiantes que elaboren una lista de los errores ortográficos que les señala el corrector ortográfico (de Word) en un determinado texto. A partir de la lista elaborada, los estudiantes deberán analizar los errores que tienen y la solución que ofrece el corrector. También se les puede solicitar que identifiquen palabras que repitan en sus escritos y busquen sinónimos en un diccionario de sinónimos y antónimos que se encuentre en Internet. El estudiantado también puede intercambiar sus borradores e identificar palabras, cuyo significado no conocen. Posteriormente pueden utilizar el diccionario de la Real Academia Española para buscar el significado de esas palabras y valorar si están usadas correcta o incorrectamente.

Esta estrategia se ejecutó con el grupo de estudiantes que cursaba Redacción y Ortografía en el Recinto de Guápiles perteneciente a la Universidad de Costa Rica. Se valoró positivamente la incorporación de estas herramientas para la revisión y edición de los textos que incluían en las bitácoras. Se consideró que las herramientas eran de fácil acceso y contribuían al uso correcto (de acuerdo con la norma) del idioma español.

\section{Conclusiones}

En la experiencia expuesta anteriormente, el análisis de bitácoras de trabajo, el análisis de los productos elaborados por las personas participantes (docentes y estudiantes), así como lo manifestado en foros, conversatorios y entrevistas realizadas permite afirmar que la implementación del aprendizaje combinado fomenta el desarrollo de las habilidades requeridas para la comunicación escrita.

Escribir es un proceso que está conformado por cuatro etapas: acceso al conocimiento, planificación del escrito, producción textual, revisión y divulgación. El acceso al conocimiento implica elegir un tema, revisar los conocimientos que se tienen sobre ese tema, buscar más información, sintetizar, establecer inferencias, en fin comprender la información, tomar posiciones, elegir argumentos para ampliar, explicar o fundamentar las ideas expuestas. A través de la estrategia Aprendo a discriminar información en internet, el estudiantado que participó en la experiencia desarrolló habilidades para elegir información confiable a partir del dominio de criterios para evaluar fuentes de información en Internet, utilización de la herramienta para búsquedas avanzadas, uso de operadores boleanos para precisar las búsquedas, uso de google académico, conocimiento sobre el significado de una dirección de Internet. Una vez que contaban con información confiable sobre un tema, se dedicaron a profundizar en la estructura 
y el significado de esa información, para esto el uso de mapas conceptuales les facilitó esa tarea, ya que les permitió entender y comprender la información que elegían. Esta herramienta les facilitó la jerarquización y establecimiento de relaciones entre conceptos. También les permitió planificar sus escritos, estableciendo conceptos y relaciones que debían desarrollar a la hora de escribir sobre determinados temas. El uso de los gestores bibliográficos contribuyó a crear, mantener, organizar y dar forma a las referencias bibliográficas de acuerdo con el estilo APA. Con la ayuda de gestores esta tarea se hizo conforme se fueron consultando los documentos y no resultó complicada ni tediosa.

Los foros electrónicos permitieron intercambiar información, opiniones, replantearse interpretaciones que habían hecho, ampliar conocimiento sobre los temas que se discutían en los foros y repensar la forma de escribir correctamente (entiéndase de acuerdo con la norma). La elaboración de wikis permitió trabajar colaborativamente, sin necesidad de coincidir en el tiempo y el espacio. Fomentó la construcción colectiva del conocimiento sobre los temas elegidos y trabajados en equipo.

Las estrategias puestas en prácticas generaron procesos auténticos de escritura y lectura que contribuyeron a la interpretación de ideas leídas, lo cual llevó a la creación de nuevas ideas y nuevos problemas sobre los cuales hablar y discutir. Esto favoreció la calidad del contenido de los escritos (argumentación, coherencia), así como al uso correcto de la ortografía, pues según manifestaron las personas participantes, el proceso de revisión y edición de texto se facilitó con el uso de los correctores ortográficos y los diccionarios en línea (ya que aunque sabían que existían no se utilizaban sistemáticamente como parte del proceso de escribir). La elaboración de bitácoras electrónicas permitió la divulgación de los escritos elaborados y motivó a estudiantes y a docentes participantes en la investigación a desarrollar su creatividad y a compartir el material escrito con otras personas, así la tarea de escribir se convirtió en algo más que cumplir con una obligación académica.

El uso de los foros electrónicos como parte de un proceso de escritura académico, permitió a la población participante detenerse a pensar en la necesidad de usar el registro adecuado, se dieron cuenta de que no era conveniente escribir en un foro electrónico con intereses académicos, de la misma manera que escribían cuando conversaban con amistades (vía mesenger, chat, mensajes de texto por celular).

La revisión de los textos resultó una tarea fácil, el formato digital facilitó esa labor, lo cual trajo consigo la elaboración de mayor cantidad de borradores y esto a su vez repercutió en la calidad de los textos escritos.

En síntesis, el aprendizaje combinado fomenta el desarrollo de las habilidades de escritura, pues Internet cuenta con herramientas que permiten potenciar la ejecución de cada una de las etapas que contempla el proceso de escribir, a saber: acceso al conocimiento, 
planificación del escrito, producción del texto, revisión y divulgación. En la figura $1^{2}$ se pueden observar gráficamente las diferentes herramientas de la Web 2.0 que pueden ser utilizadas para apoyar los procesos mencionados.

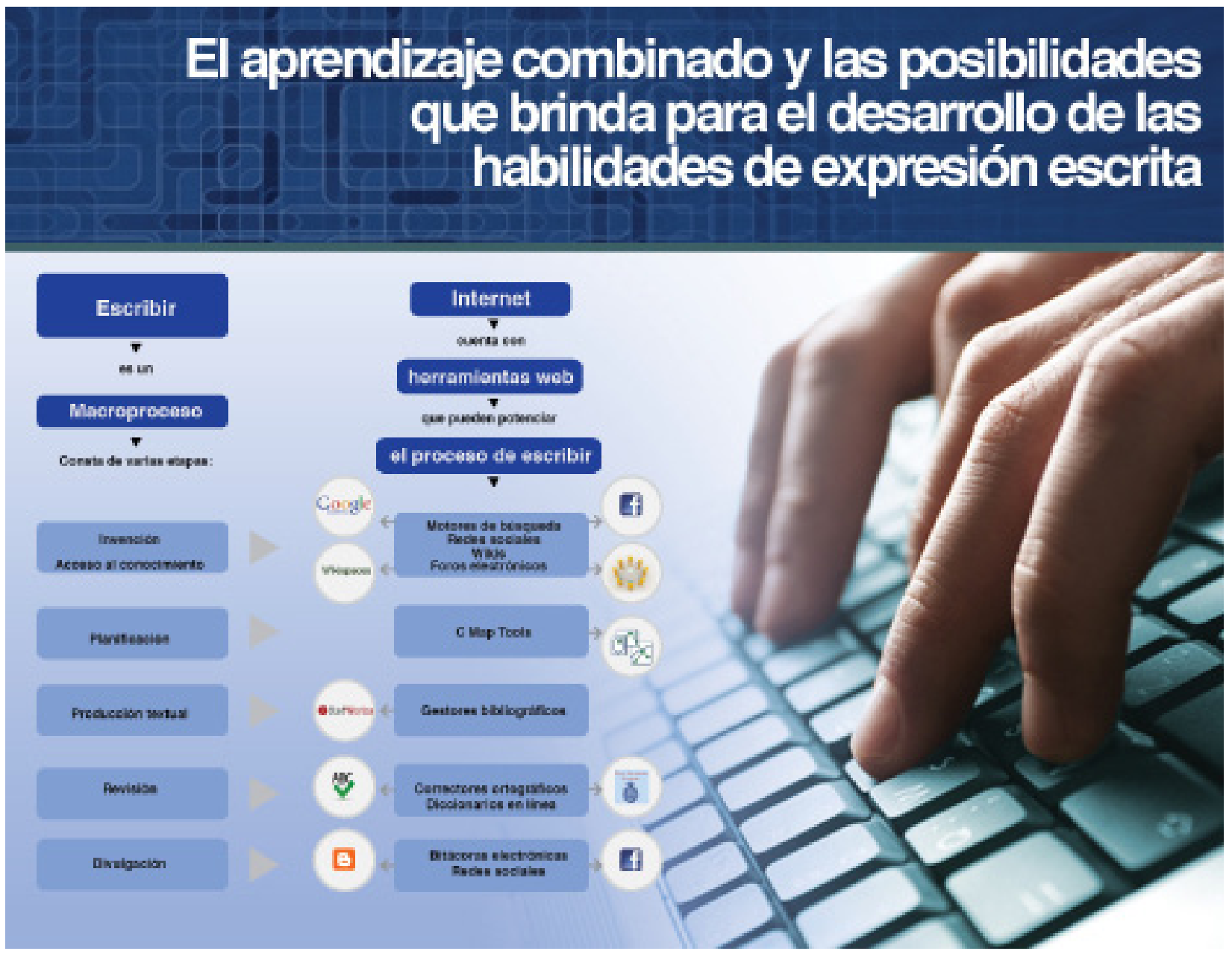

\section{Referencias}

Álvarez, T. y Ramírez, R. (2006). Teorías o modelos de producción de textos en la enseñanza y el aprendizaje de la escritura. Didáctica (Lengua y Literatura), 18, 29-60. Recuperado de http://revistas.ucm.es/index.php/DIDA/article/view/DIDA0606110029A/19144

Argüello, B., Mora, L. y Quirós, M. A. (2012). Utilización de las tecnologías de la información y la comunicación para el mejoramiento de la escritura del estudiantado de tercer ciclo de la educación general básica (Tesis de licenciatura inédita). San José, Universidad de Costa Rica.

\footnotetext{
La imagen es propiedad de la autora del artículo, y fue elaborado a partir de los insumos que generó la investigación realizada. Se diseñó en el departamento de Publicaciones del Instituto Tecnológico de Costa Rica (TEC); la fotografía que ilustra la imagen fue tomada del banco nominado "Shutterstoc" y que el TEC adquirió dando la posibilidad de reproducir un determinado número de descargas diarias, las cuales podían usarse para ilustraciones siempre y cuando no se superen las 20.000 impresiones que contengan la imagen.
} 
Coaten, N. (octubre, 2003). Blended e-learning [Aprendizaje combinado]. Educaweb. com, 69. Consultado en http://www.educaweb.com/esp/servicios/monografico/ formacionvirtual/1181076-a.html

Henao, O. (2006). Evidencias de la investigación sobre el impacto de las tecnologías de la información y comunicación en la enseñanza de la lecto-escritura. Revista Educación y Pedagogía. 18(44), 71-87. Consultado en http://aprendeenlinea.udea.edu.co/revistas/ index.php/revistaeyp/article/viewFile/6073/5479

Marsh II, G. E., McFadden, A. C. y Price, B. J. (Winter, 2003) Blended Instruction: Adapting Conventional Instruction for Large Classes [Aprendizaje combinado: Adaptación de instrucción convencional para grandes clases]. Online Journal of Distance Learning Administration, 6(4). Recuperado de http://www.westga.edu/ distance/ojdla/winter64/ marsh64.ht

Sánchez, C. (2005). Los problemas de redacción de los estudiantes costarricenses: Una propuesta de revisión desde la lingüística del texto. Filología y Lingüística, 31(1), 267-295. Recuperado de http://www.vinv.ucr.ac.cr/latindex/rfl-31-1/rfl-31-1-15.pdf

Sandín, M. P. (2003). Investigación culitativa en educación. Fundamentos y tradiciones. Madrid: McGraw-Hill. Recuperado de http://www.postgrado.unesr.edu.ve/acontece/es/ todosnumeros/num09/02 05/capitulo 7 de sandin.pdf

Solano, O. L. (septiembre de 2010). Las posibilidades que brinda el aprendizaje combinado (modalidad presencial y no presencial apoyado por el uso de Internet) para el desarrollo de las destrezas de comunicación escrita. En Congreso Iberoamericano de Educación (pp. 1-15). Buenos Aires, República de Argentina. Recuperado de http://www.chubut.edu.ar/ descargas/secundaria/congreso/TICEDUCACION/R1006 Solano.pdf

Tinajero, E. (agosto, 2006). Internet y computadoras en educación: Una visión sociocultural. Apertura: Revista de Innovación Educativa, 6(4), 90-105. Recuperado de http://www. redalyc.org/pdf/688/68800408.pdf

\section{Cómo citar este artículo en APA:}

Solano, O. L. (2013). El aprendizaje combinado y el desarrollo de las habilidades requeridas para la comunicación escrita. Revista Electrónica Educare, 17(3), 293-313. Recuperado de http://www.revistas.una.ac.cr/index.php/EDUCARE/issue/current

Nota: Para citar este artículo en otros sistemas puede consultar el hipervínculo "Como citar el artículo" en la barra derecha de nuestro sitio web:

http://www.revistas.una.ac.cr/index.php/EDUCARE/index 